\title{
Shift multiplexing with spherical reference waves
}

\author{
George Barbastathis, Michael Levene, and Demetri Psaltis
}

\begin{abstract}
Shift multiplexing is a holographic storage method particularly suitable for the implementation of holographic disks. We characterize the performance of shift-multiplexed memories by using a spherical wave as the reference beam. We derive the shift selectivity, the cross talk, the exposure schedule, and the storage density of the method. We give experimental results to verify the theoretical predictions.

Key words: Shift multiplexing, holographic three-dimensional disks, cross talk, exposure schedule, surface storage density. (1) 1996 Optical Society of America
\end{abstract}

\section{Introduction}

Shift multiplexing was proposed in Ref. 1 as a holographic storage method particularly suitable for holographic three-dimensional (3-D) disks. ${ }^{2,3}$ The design of a shift-multiplexed disk is shown in Fig. 1. The information to be stored on the disk is imprinted on a plane-wave signal beam that illuminates a spatial light modulator (SLM). Data can be analog or digital, depending on the application. The reference is a spherical wave produced by a lens of high numerical aperture (NA). The data are stored on the disk as a hologram recorded by the interference of the signal and the spherical reference. Alternatively, the reference can be a one- or two-dimensional fan of plane waves of arbitrary relative phases.

The nonplanar phase front of the reference beam allows one to multiplex and retrieve holograms selectively simply by translating the disk relative to the recording head, as shown in Fig. 1. The shift selectivity, i.e., the translation required for resolving shift-multiplexed holograms, is typically of the order of a few micrometers, much less than the transverse size of the holograms (the latter is typically a few millimeters). In this way multiple overlapping holograms are superimposed. To reconstruct holograms that belong to the same track selectively, the disk is

The authors are with the California Institute of Technology, Mail-Stop 136-93, Pasadena, California 91125: G. Barbastathis and D. Psaltis are with the Department of Electrical Engineering and M. Levene is with the Department of Computation and Neural Systems.

Received 28 July 1995; revised manuscript received 13 November 1995.

0003-6935/96/142403-15\$10.00/0

(C) 1996 Optical Society of America rotated relative to the stationary head. The head needs to move only in the radial direction to access different tracks on the disk. No additional multiplexing mechanism is needed. Because both disk rotation and radial head translation are an integral part of the optical disk configuration, a shiftmultiplexed disk is a simple implementation.

In this paper we concentrate on the implementation of shift multiplexing by the use of a spherical wave reference. We theoretically explain the shiftselectivity properties of volume holograms recorded with spherical reference beams and present experimental selectivity curves. We also give experimental results on cross talk between holograms superimposed by the shift-multiplexing method and show that cross talk behaves approximately the same as in the case of angle-multiplexed holograms. We address the issue of dynamic range for shift-multiplexed holograms in photorefractive materials and give two alternative exposure schedules, sequential and interleaved recording. We demonstrated the sequential technique by storing 600 shift-multiplexed holograms in $\mathrm{LiNbO}_{3}$.

The storage density of angle- and wavelengthmultiplexed holographic 3-D disks was derived in Ref. 3. It was shown that uniformity considerations for the edges of the stored holograms cause the density to peak at a theoretical maximum of 117.2 bits $/ \mu^{2}$ (for typical SLM parameters and optical apertures) for a $16.7-\mathrm{mm}$-thick $\mathrm{LiNbO}_{3}$ disk, with four symmetric reference angles used for recording. In Section 5 we present the corresponding derivation for shift multiplexing. We show that the density of a shift-multiplexed disk increases monotonically with thickness and eventually saturates. 


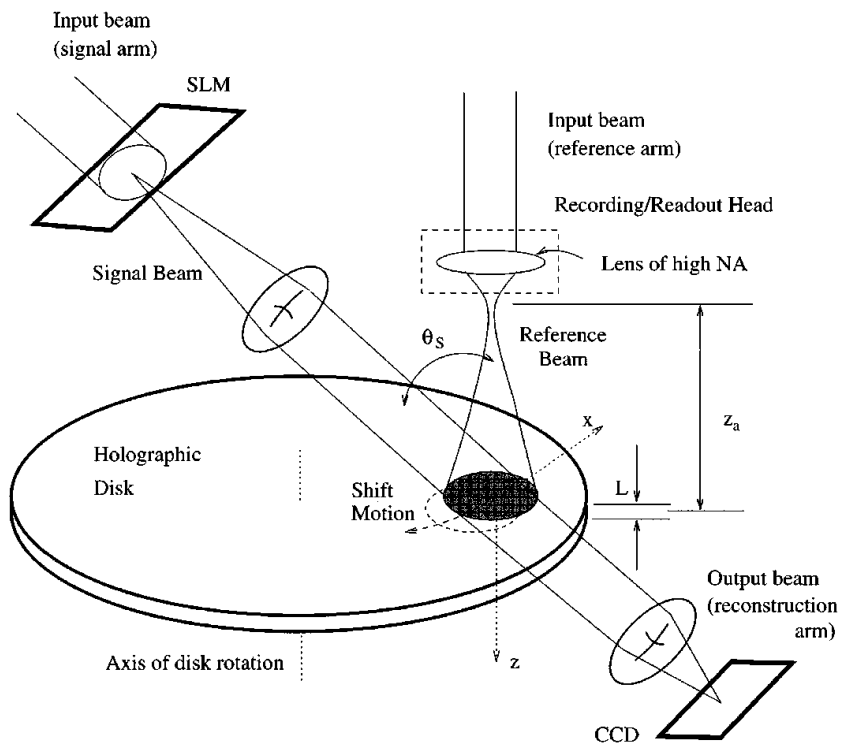

Fig. 1. Holographic disk implemented with shift multiplexing. SLM, spatial light modulator; NA, numerical aperture.

\section{Volume Holography with Spherical Reference Beams}

The use of spherical reference beams in volume holography was treated in Refs. 4-7. In Ref. 4 a spherical reference was used for a holographic correlator, and the shift invariance curves were obtained theoretically and experimentally. Here we use a similar approach to derive the shift selectivity of shift-multiplexed memories.

The geometry for shift multiplexing by the use of spherical waves is shown in Fig. 2. The hologram is recorded in the region $|z|<L / 2$ and is assumed to be

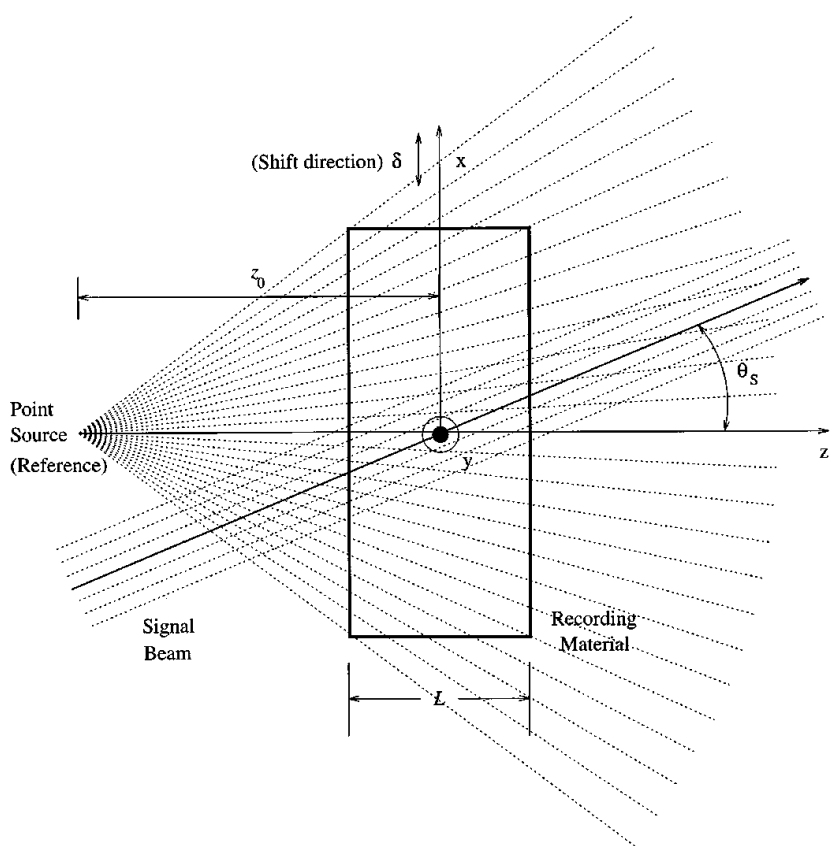

Fig. 2. Geometry for shift multiplexing by the use of a spherical reference wave. infinite in the transverse directions $x, y$. The spherical reference wave is produced by a spherical lens of high NA. The focus is located at $z=-z_{0}$. The expression for the reference beam in the chosen system of coordinates and under the paraxial approximation is

$$
\begin{aligned}
R(x, y, z)= & \frac{1}{j \lambda\left(z+z_{0}\right)} \exp \left(j 2 \pi \frac{z+z_{0}}{\lambda}\right) \\
& \times \exp \left[j \pi \frac{x^{2}+y^{2}}{\lambda\left(z+z_{0}\right)}\right] .
\end{aligned}
$$

We consider a plane-wave component of the signal beam incident upon the $x-z$ plane, making angle $\theta_{S}$ with the $z$ axis, which is expressed as

$$
S(x, z)=\exp \left[j 2 \pi u_{S} \frac{x}{\lambda}+j 2 \pi\left(1-\frac{u_{S}^{2}}{2}\right) \frac{z}{\lambda}\right],
$$

where $u_{S} \equiv \sin \theta_{S} \approx \theta_{S} \ll 1$ (paraxial approximation). If we neglect the variation of the modulation depth throughout the hologram because of the defocusing of the spherical wave, then the hologram can be expressed by the term $R^{*}(x, y, z) S(x, z)$ in the resulting interference pattern. We now consider the expression for the field diffracted from a thin layer of the hologram located at $z$ by using a displaced reference beam $R(x-\delta, y, z)$ :

$$
\begin{aligned}
R(x-\delta, y, z) R^{*}(x, y, z) S(x, z) & \\
=\exp [ & \left.-j \pi \frac{2 \delta x}{\lambda\left(z+z_{0}\right)}\right] \exp \left[j \pi \frac{\delta^{2}}{\lambda\left(z+z_{0}\right)}\right] \\
\quad & \exp \left(j 2 \pi u_{S} \frac{x}{\lambda}\right) \exp \left[j 2 \pi\left(1-\frac{u_{S}{ }^{2}}{2}\right) \frac{z}{\lambda}\right] .
\end{aligned}
$$

The signal beam is reconstructed if $\delta=0$. For $\delta \neq 0$, the first term in Eq. (3) causes the reconstruction to deviate angularly with respect to the original signal $S(x, z)$ by an amount

$$
\Delta \theta_{S} \approx \frac{\delta}{\left(z+z_{0}\right) \cos \theta_{S}} .
$$

Because this angular deviation has a $z$ dependence, reconstructions coming from successive thin slices of the hologram are phase mismatched. The amount of shift $\delta$ required for exactly canceling the reconstruction is calculated in Appendix A (under the paraxial, Born, and constant modulation-depth approximations), and it is given by

$$
\delta_{\text {Bragg }}=\frac{\lambda z_{0}}{L u_{S}} .
$$

It is interesting that, in the geometry of Fig. 2, if the reference were a plane wave incident along the $z$ axis instead of the spherical wave, then the angular 
selectivity would be

$$
\Delta \theta=\frac{\lambda}{L \tan \theta_{S}} \approx \frac{\lambda}{L u_{S}} .
$$

Thus we obtain the useful formula

$$
\delta_{\text {Bragg }}=z_{0} \Delta \theta .
$$

The finite spot size $\Delta x=\lambda / 2(\mathrm{NA})$ of a truncated spherical wave introduces ambiguity in the location of the point source with respect to the hologram. This ambiguity must be added to the shift selectivity, giving the final expression

$$
\begin{aligned}
\delta & =\delta_{\mathrm{Bragg}}+\Delta x \\
& =\frac{\lambda z_{0}}{L \tan \theta_{S}}+\frac{\lambda}{2(\mathrm{NA})} .
\end{aligned}
$$

So far we have assumed a holographic medium with an index of refraction equal to 1 . Unless an index-matching liquid is used, the change in refraction index $n_{0}$ at the interface of the holographic material causes the apparent location of the point source (as seen by an observer inside the holographic medium) to move away from the hologram. If we let $z_{a}$ denote the distance of the point source from the center of the holographic material, measured in air, then the apparent $z_{0}$ relates to $z_{a}$ (paraxially) as

$$
z_{0}-\frac{L}{2}=n_{0}\left(z_{a}-\frac{L}{2}\right)
$$

Therefore the modified selectivity equation is

$$
\delta=\frac{\lambda_{0}\left[z_{a}-\left(1-\frac{1}{n_{0}}\right) \frac{L}{2}\right]}{L \tan \theta_{S^{\prime}}}+\frac{\lambda_{0}}{2(\mathrm{NA})} .
$$

where $\lambda_{0}$ denotes the wavelength of light in vacuum and $\theta_{S}{ }^{\prime}$ is the angle of incidence of the signal inside the material, determined from Snell's law.

The experimental geometry used for all the shiftmultiplexing experiments described in this paper is shown in Fig. 3. The experimental parameters were $\lambda_{0}=488 \mathrm{~nm}, L=4.5 \mathrm{~mm}, \theta_{S}=40^{\circ}$ (measured outside the crystal), $z_{a}=1 \mathrm{~cm}$ (distance from focus of the spherical reference to the center of the crystal, measured in air), and NA $=0.6$. The recording material $\left(\mathrm{Fe}\right.$-doped $\left.\mathrm{LiNbO}_{3}\right)$ has an index of refraction of $n_{0} \approx 2.24$. The signal was a chessboard pattern, recorded as a Fresnel-region hologram. The size of each square in the chessboard at the SLM plane was approximately $0.5 \mathrm{~mm}$. For the parameters used in the experiment, Eq. (10) yields $\delta=3.58$ $\mu \mathrm{m}$. The experimental selectivity curve is shown in Fig. 4. The first null occurred at approximately $3.7 \pm 0.2 \mu \mathrm{m}$ (the margin of error is mainly due to stage inaccuracy and backlash), deviating by $3.6 \%$ from the theoretical prediction.

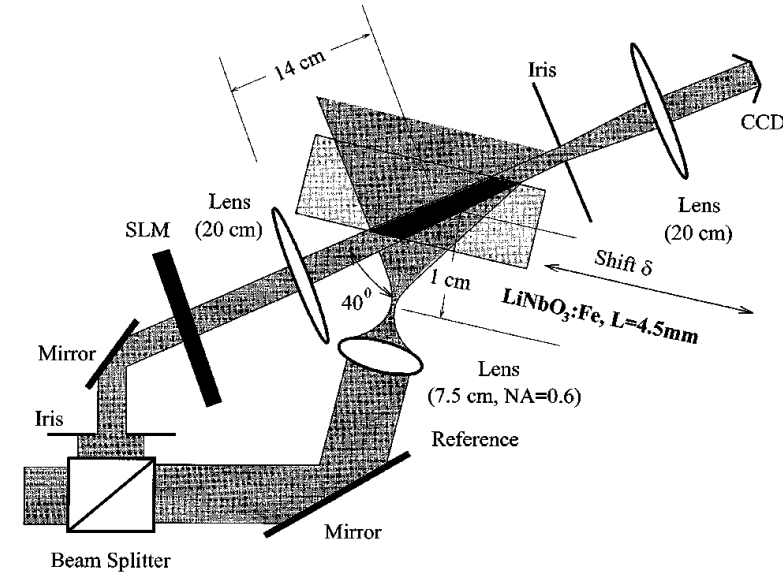

Fig. 3. Experimental setup for the demonstration of shift multiplexing (not drawn to scale).

\section{Cross Talk in Shift Multiplexing}

The approximate theory presented in Appendix A predicts that the diffraction efficiency $\eta$ of spherical volume holograms as a function of shift has nulls at integer multiples of $\delta_{\text {Bragg. }}$. This holds for the ideal situation of a hologram that is infinite in the transverse directions, recorded with a spherical wave of zero spot size as reference and a plane wave as signal. We also neglected the variable modulationdepth effects that were due to the variation in intensity of the reference and the signal throughout the volume of the hologram. Finally, the calculation was performed for a single signal component incident at $\theta_{S}$. In general, the signal occupies a

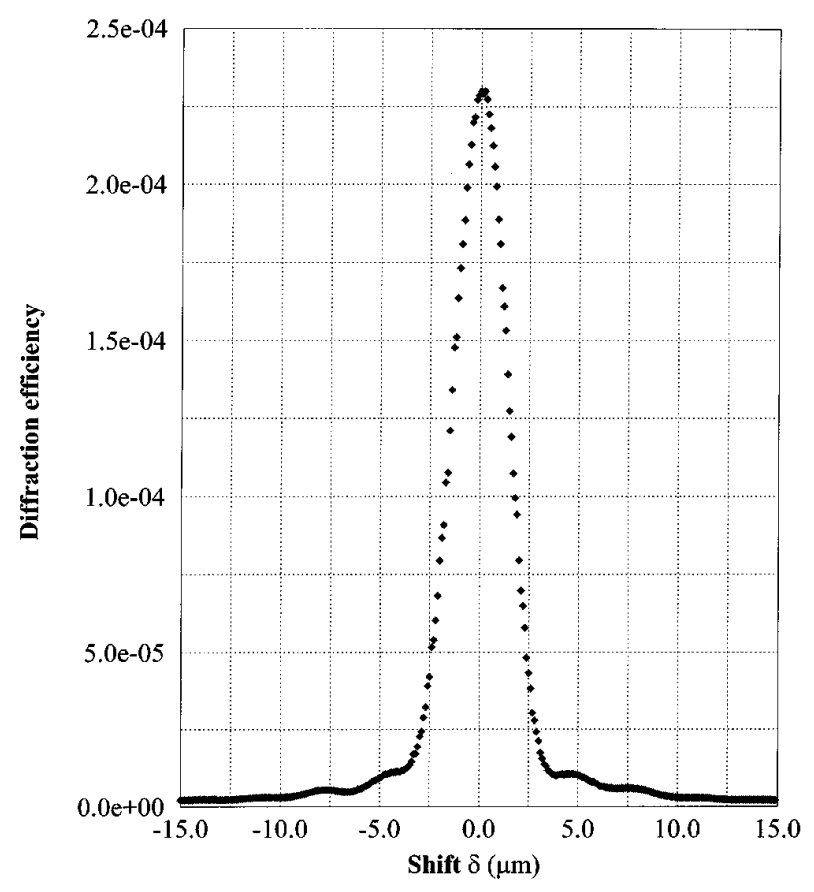

Fig. 4. Experimental selectivity curve (diffraction efficiency $\eta$ versus shift $\delta$ ). The parameters of the experiment are shown in Fig. 3. 
finite-size bandwidth in reciprocal space, hence each component Bragg mismatches at different $\delta$.

In this section, we develop a theoretical model for the cross talk induced by the finite signal bandwidth in the case of shift multiplexing in the Fourier plane. In the calculation we drop the dependence of the selectivity on the NA (i.e., the $\Delta x$ correction). The assumption of an infinite spherical wave for shift multiplexing is equivalent to assuming an infinite plane-wave reference for other methods, as was done in calculations of cross talk for angle, ${ }^{8}$ wavelength, ${ }^{9,10}$ and phase-code ${ }^{11}$ multiplexing in the Fourier plane and for image-plane holograms. ${ }^{12} \mathrm{We}$ show that, under these assumptions, the results for shift multiplexing are consistent with the anglemultiplexing analysis. Then we characterize the cross talk experimentally and compare the results with the theory.

Consider the Fourier-plane geometry of Fig. 5. Let $f_{m}(\xi, \eta), m=1, \ldots, M$ denote the pattern stored as the $m$ th page. $M$ is the maximum number of overlapping pages on any location. The signal corresponding to the $m$ th hologram is expressed as

$$
\begin{aligned}
& S_{m}(x, y, z) \\
& =\int_{-\infty}^{+\infty} \int_{-\infty}^{+\infty} \mathrm{d} \xi \mathrm{d} \eta f_{m}(\xi, \eta) \\
& \quad \times \exp \left[-j 2 \pi \frac{\xi}{\lambda F}\left(-\sin \theta_{S} z+\cos \theta_{S} x\right)-j 2 \pi \frac{\eta y}{\lambda F}\right] \\
& \quad \times \exp \left[j \frac{2 \pi}{\lambda}\left(1-\frac{\xi^{2}+\eta^{2}}{2 F^{2}}\right)\left(\cos \theta_{S} z+\sin \theta_{S} x\right)\right] .
\end{aligned}
$$

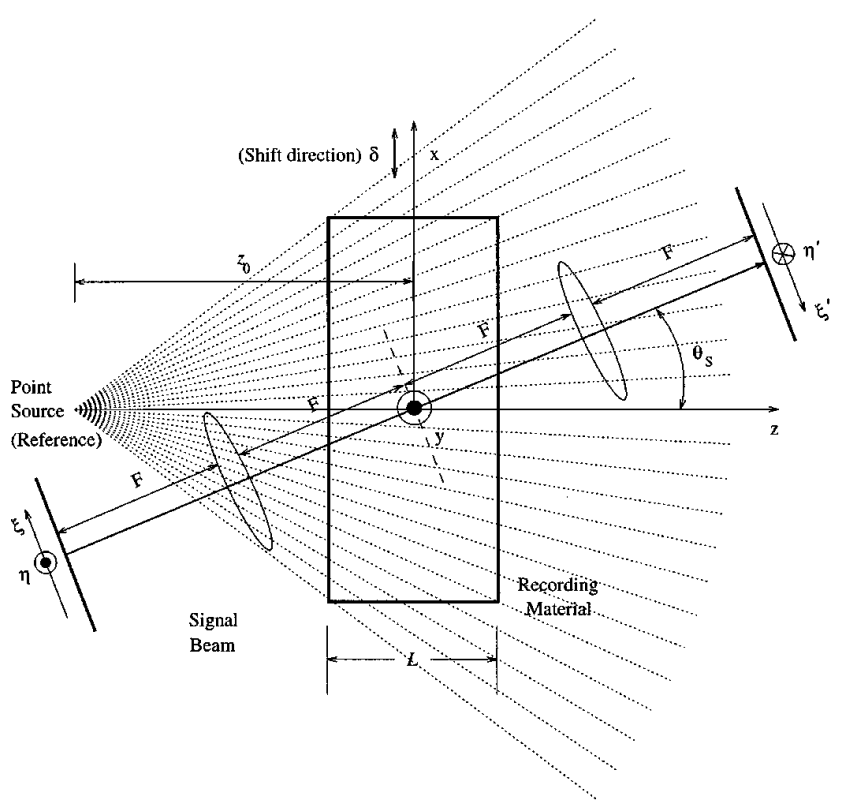

Fig. 5. Geometry for the theoretical calculation of cross talk in shift multiplexing by the use of a spherical reference wave.
To reconstruct hologram $m^{\prime}$, the recording area is illuminated by a spherical beam displaced by $m^{\prime} \delta$ :

$$
\begin{aligned}
\mathscr{E}_{i}\left(m^{\prime}\right)= & \frac{1}{j \lambda\left(z+z_{0}\right)} \exp \left(j 2 \pi \frac{z+z_{0}}{\lambda}\right) \\
& \times \exp \left[j \pi \frac{\left(x-m^{\prime} \delta\right)^{2}+y^{2}}{\lambda\left(z+z_{0}\right)}\right] .
\end{aligned}
$$

We obtain the diffracted field by using the theory of Appendix A with the paraxial approximation $\sin \theta_{S} \equiv u_{S} \ll 1, \cos \theta_{S} \approx 1-u_{S}^{2} / 2$ and neglecting refraction. A lengthy but straightforward calculation yields for the detector plane the following expression:

$$
\begin{aligned}
\mathscr{E}_{m^{\prime}}\left(\xi^{\prime}, \eta^{\prime}\right) \approx & \sum_{m=0}^{M-1} f_{m}\left[\xi^{\prime}+\frac{\left(m-m^{\prime}\right) \delta}{z_{0}} F, \eta^{\prime}\right] \\
& \times \operatorname{sinc}\left[\frac{\left(m-m^{\prime}\right) \delta L}{\lambda z_{0}}\left(u_{S}-\frac{\xi^{\prime}}{F}\right)\right] .
\end{aligned}
$$

A straightforward calculation, along the lines of Ref. 8 , shows that a similar expression holds approximately for the cross talk in the geometry of Fig. 5 (in the paraxial approximation) if we replace the spherical reference wave with a plane wave parallel to the $z$ axis and perform angle multiplexing instead of shift multiplexing. A significant difference between the cases of shift and the exact solution for angle multiplexing is that, in the former, symmetry makes cross talk depend on the difference $m-m^{\prime}$ only.

When hologram $m^{\prime}$ is reconstructed, the fact that the remaining multiplexed holograms were recorded displaced by a multiple of the shift selectivity $\delta$ guarantees only that their central component, i.e., the central pixel $\xi^{\prime}=0$, will be Bragg mismatched. All other locations in the multiplexed images still diffract weakly, because their shift selectivity is given by Eq. (5) with $u_{S}-\xi^{\prime} / F$ rather than with $u_{S}$. These contributions appear as cross talk around the noise-free central pixel.

Let us assume that a large number $M$ of Fourierplane holograms are shift multiplexed and are separated by $p$-shift Bragg nulls, i.e., the relative translation between successive recordings is $p \delta$, where $\delta$ is the shift selectivity. Under the image statistics assumed in Refs. 8, 10, and 11, the expected value of the cross-talk noise power is given by the expression

$$
P_{X N} \approx \sum_{m=0}^{M-1} \operatorname{sinc}^{2}\left[p\left(m-m^{\prime}\right)\left(1-\frac{\xi^{\prime}}{u_{S} F}\right)\right],
$$

where the signal power was taken to be equal to 1 . If

$$
\frac{p\left|\xi^{\prime}\right|}{u_{S} F} \ll 1, \quad M \rightarrow \infty,
$$

then the summation can be carried out analytically 
and yields

$$
P_{X N} \approx \frac{\left|\xi^{\prime}\right|}{2 p u_{S} F} .
$$

Therefore, at pixels lying close to the carrier $u_{S}$, the noise increases linearly with distance from the image center and is inversely proportional to the null order $p$.

Theoretical plots of exact relation (14) are given in Fig. 6. As the pixel value increases, relation (15) is violated and the noise pattern becomes asymmetric. Pixels with large positive values are closer to the $z$ axis and suffer from higher noise. The same curves hold approximately for angle multiplexing in the off-axis geometry if the same parameters (including the number of holograms $M$ ) are used.

To characterize the cross-talk effects for shiftmultiplexed volume holograms recorded with spherical reference beams, we performed the following experiment: We stored 20 holograms of rotated versions of the same chessboard pattern in 21 shiftmultiplexed positions, leaving position 11 blank. Therefore excess light measured in the location of hologram 11 is due to cross-talk contributions from the neighboring holograms. The shift separation between adjacent holograms was chosen to be equal to $\delta, 2 \delta, 3 \delta$, and $4 \delta$ (i.e., $p=1,2,3,4$, respectively), where for $\delta$ we used the experimentally determined value of $3.7 \mu \mathrm{m}$. The holograms were stored in the Fresnel region. The cross-talk theory developed for Fourier-plane holograms also applies to Fresnel

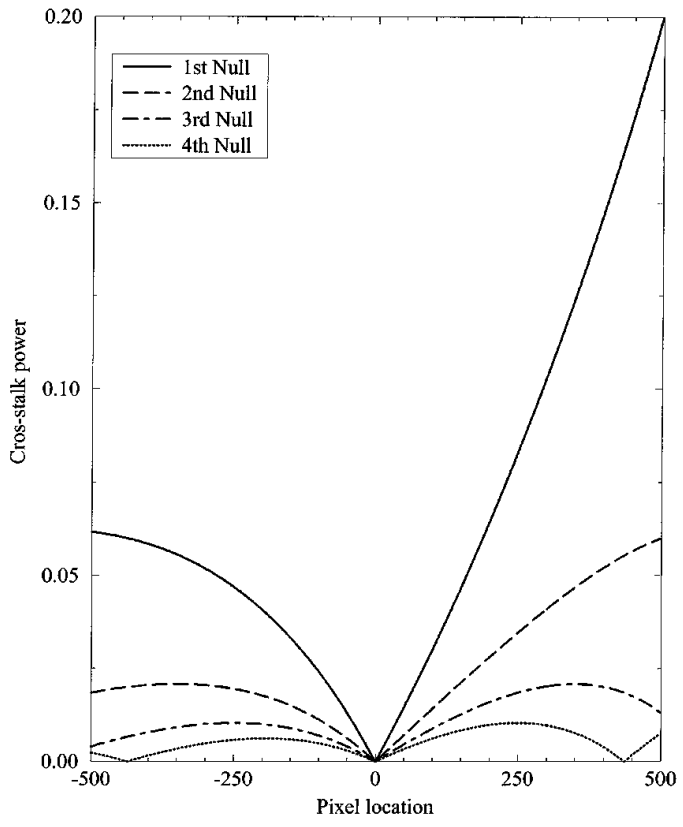

Fig. 6. Theoretical plots of expected cross-talk power versus pixel location for Fourier-plane shift-multiplexed holograms. The parameters used for the plots were hologram thickness $L=1$ $\mathrm{mm}$, angle of incidence of the signal $\theta_{S}=20^{\circ}$, wavelength $\lambda=488$ $\mathrm{nm}$, focal length $F=5 \mathrm{~cm}$, and pixel size $b=10 \mu \mathrm{m}$.
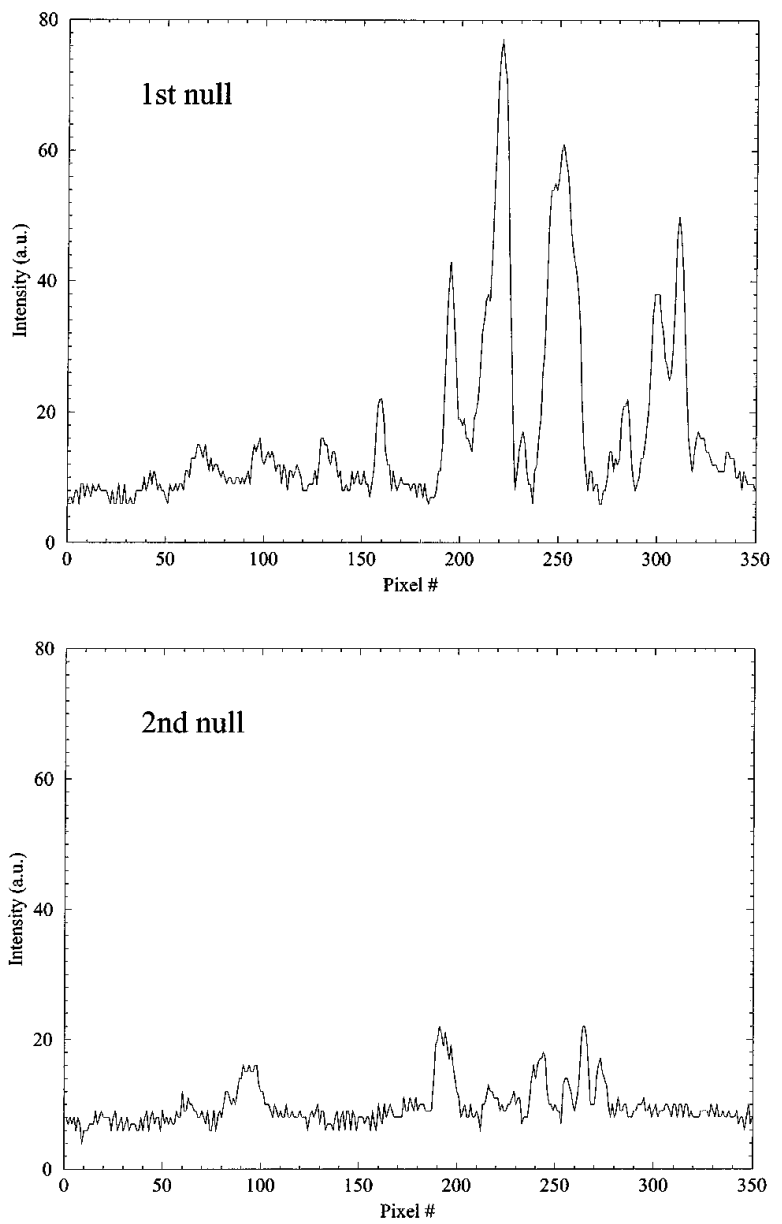

Fig. 7. Cross sections of the diffracted pattern at shift location 11 (originally left blank). The upper plot is for multiplexing holograms in the first Bragg null and the lower plot for the second null. The units on both axes are arbitrary, but horizontal and vertical scales are the same in both plots.

holograms recorded anywhere between the two lenses in a $4 F$ imaging system.

Behavior consistent with that predicted in Fig. 6 is observed in Fig. 7, where the cross section of the reconstruction of location 11 is plotted (in the absence of cross talk, this would contain only scatter and detector noise contributions). The asymmetry predicted in approximation (13) is evident for storage in the first null. In the case of using the second null, the noise power decreases considerably and the assymetry becomes less pronounced, in agreement with the theoretical curves of Fig. 6.

The signal-to-noise ratio (SNR) results are given in Fig. 8 for the cases of a single hologram and 21 multiplexed holograms. In the case of a single hologram, we calculated the SNR by measuring the spatially averaged diffracted power from the hologram at zero translation and dividing by the diffracted power at shifts equal to $\delta, 2 \delta, 3 \delta$, and $4 \delta$. For the multiple holograms, we calculated the SNR by dividing the diffraction efficiency at location 10 


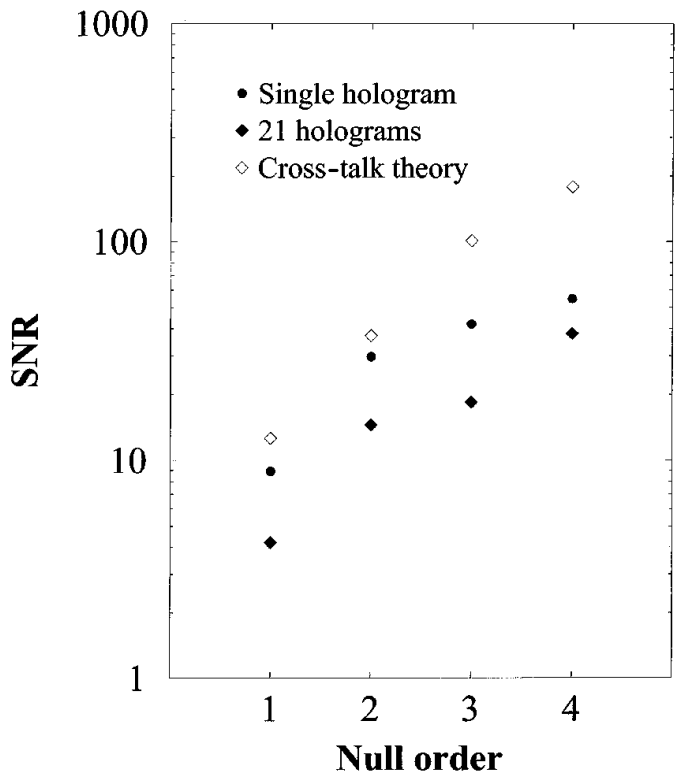

Fig. 8. SNR versus null order $p$ (in multiples of $\delta=3.7 \mu \mathrm{m}$ ) for two experiments: single hologram and 21 holograms. Shown also is the theoretical SNR prediction for the maximum number $M$ of allowable shift-multiplexed holograms at the respective null orders.

with the diffraction efficiency at location 11 (empty slot) for the four cases of null separation.

In the same plot we also give the theoretical values of the ratio between the expected total signal power and the expected total noise power for each case of null separation. The three curves show the same qualitative behavior, although there is a noticeable discrepancy between the theoretical and the experimental values of cross-talk-induced SNR. The saturating behavior of the experimental data indicates that the discrepancy is mainly due to noise sources unrelated to cross talk, such as scatter noise and multiple reflections off the uncoated crystal surfaces. In addition, small contributions are present from cross-talk sources, such as finite NA and variable modulation depth that we neglected in the theory.

\section{Exposure Schedule for Shift Multiplexing in Photorefractive Materials}

The diffraction efficiency $\eta$ of holograms recorded in diffusion-dominated photorefractive materials is described as a function of the recording time $t$ by a saturating exponential of the form (see, e.g., Ref. 13)

$$
\eta(t)=\eta_{0}\left[1-\exp \left(-t / \tau_{w}\right)\right]^{2},
$$

where $\eta_{0}$ is the saturation diffraction efficiency and $\tau_{w}$ is the recording time constant. On the other hand, when a hologram of strength $\eta_{1}$ is illuminated, it decays exponentially as

$$
\eta(t)=\eta_{1} \exp \left(-2 t / \tau_{e}\right)
$$

where $\tau_{e}$ is the erasure time constant. The param- eters $\tau_{w}$ and $\tau_{e}$ depend on the geometry, the total exposure power, the modulation depth, and the absorption coefficient. In this paper we do not do a detailed calculation of the time constants, but we assume that the exponential models of Eqs. (17) and (18) hold, and we determine the value of $\tau_{e}$ experimentally.

In multiplexing techniques based on recording over the same spot (e.g., angle multiplexing), holograms recorded early are erased by their successors. The first holograms are erased more, thus they must be initially stronger; this requirement was used in Refs. 14-16 to derive hologram recording times as a function of hologram order. This function is referred to as exposure schedule. The exposure times depend on $\tau_{w}, \tau_{e}$, and the number of holograms $M$.

We now describe an exposure schedule for shiftmultiplexed holograms that we call sequential recording. With this method, shift-multiplexed holograms are recorded when the disk is rotated by an angle $\phi_{\text {disk }}$ sufficient to produce translation equal to the shift selectivity $\delta$ between successive exposures. Let $R$ be the radius of the track being recorded. Then $\phi_{\text {disk }}$ is given by

$$
\phi_{\text {disk }}=\frac{\delta}{R}=\frac{\lambda z_{0}}{R L \tan \theta_{S}}+\frac{\lambda}{2 R(\mathrm{NA})} .
$$

Figure 9(a) shows how the sequential exposure schedule evolves in time and space. $M$ is the number of shift-multiplexed holograms that overlap within one spot. It is equal to the hologram aperture along the shift direction divided by $\delta$ (see Section 5 for a derivation). The total number of holograms fitting in the track is given by

$$
N=\frac{2 \pi R}{\delta} .
$$

A hologram is erased by its neighbors that start to its right and overlap vertically in the plot; thus $\mathrm{A}_{m}$ is erased by $\mathrm{A}_{m+1}, \ldots, \mathrm{A}_{m+M-1}$ but not by the subsequent holograms. This is true for all indices $m$ running from $M+1$ to $N-M$. Holograms $\mathrm{A}_{1}, \ldots, \mathrm{A}_{M}$ will be further erased by holograms $\mathrm{A}_{N-M+1}, \ldots, \mathrm{A}_{N}$ when the disk completes one full revolution, whereas holograms $\mathrm{A}_{N-M+1}, \ldots, \mathrm{A}_{N}$ will be erased less than the other holograms. Neglecting these edge effects, all other holograms are erased in the same manner; hence their diffraction efficiencies are equalized if they are recorded with the same exposure time $t_{0}$.

A consequence of the sequential approach is transverse nonuniformity, as shown in Fig. 9(b). Consider any hologram $\mathrm{A}_{m}$, except for the first $M$ and the last $M$. The diffraction efficiency of $\mathrm{A}_{m}$ immediately after recording is given by

$$
\eta^{1}=\eta_{0}\left[1-\exp \left(-t_{0} / \tau_{w}\right)\right]^{2}
$$

The next hologram in the sequential schedule is 

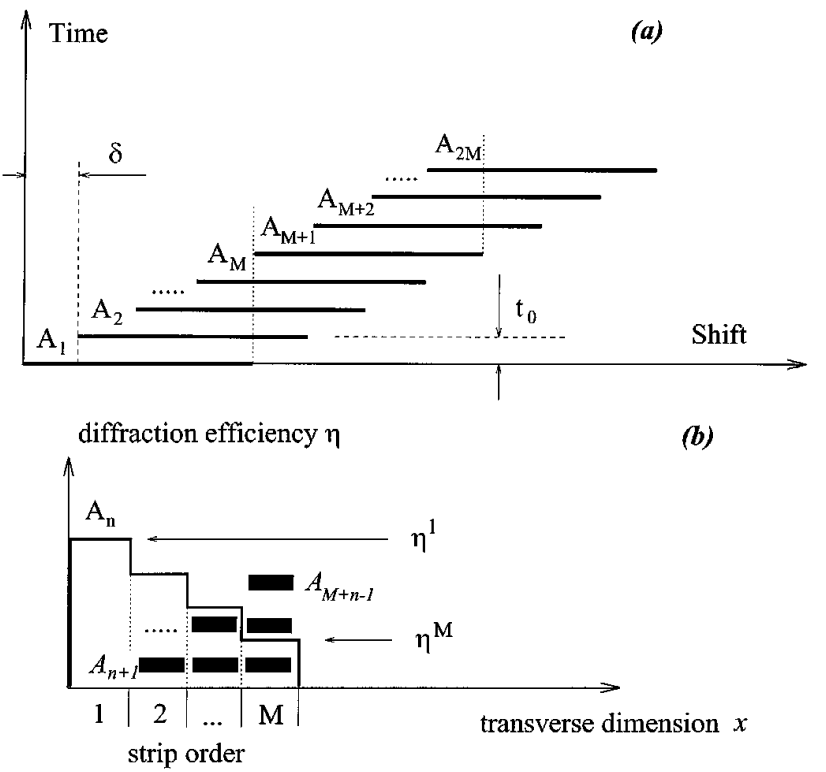

Fig. 9. (a) Exposure schedule for sequential recording. Horizontal axis is shift, vertical is recording time. Bars $A_{1}, A_{2}, \ldots, A_{2 M}$ denote holograms; the index corresponds to the location on the disk; the horizontal location of a hologram in the graph denotes its shift with respect to the origin (left edge of the first hologram $A_{1}$ ), and the vertical location the beginning of its exposure in the schedule. The horizontal separation is equal to the shift selectivity $\delta$; the vertical separation is equal to the constant exposure time $t_{0}$ (see text). (b) Nonuniform erasure of hologram $\mathrm{A}_{m}$ by its successors $\mathrm{A}_{m+1}, \ldots, \mathrm{A}_{m+M-1}$. The diffraction-efficiency curve follows the profile of $\mathrm{A}_{m}$ after the recording of all its shift-multiplexed neighbors is complete (see also Appendix B and Fig. 14).

$\mathrm{A}_{m+1}$, and it is recorded after shifting by $\delta$. Thus it will erase $\mathrm{A}_{m}$ for a time $t_{0}$, except for a strip of width $\delta$, which is denoted as strip 1 in Fig. 9(b); this strip will retain diffraction efficiency $\eta^{1}$. In general, after the end of the recording process, strip $l$ of any hologram will have reached the diffraction efficiency of

$$
\eta^{l}=\eta_{0}\left[1-\exp \left(-t_{0} / \tau_{w}\right)\right]^{2} \exp \left[-2(l-1) t_{0} / \tau_{e}\right] .
$$

Maximum erasure is suffered by strip $M$. The diffraction efficiency $\eta^{M}$ of this strip is maximized if we choose

$$
t_{0}=\tau_{w} \ln \left[1+\frac{\tau_{e}}{(M-1) \tau_{w}}\right] \approx \frac{\tau_{e}}{M}
$$

and is given by

$$
\begin{aligned}
\eta^{M} & =\eta_{0}\left[\frac{\tau_{e}}{\tau_{\mathrm{e}}+(M-1) \tau_{w}}\right]^{2}\left[1+\frac{\tau_{e}}{(M-1) \tau_{w}}\right]^{-2(M-1) \tau_{w} / \tau_{e}} \\
& \approx \eta_{0} \frac{\tau_{e}^{2} e^{-2}}{M^{2} \tau_{w}{ }^{2}}
\end{aligned}
$$

The approximations hold for $M \gg 1$. We now define the average diffraction efficiency of the nonuniform holograms as

$$
\eta_{\mathrm{av}}=\frac{\int \eta(x) \mathrm{d} x}{\int \mathrm{d} x},
$$

where the integrals are along the aperture of the holograms in the shift direction. This results in

$$
\begin{aligned}
\eta_{\mathrm{av}}= & 4 \frac{\eta_{0}}{M} \exp \left[-t_{0}\left(\frac{1}{\tau_{w}}+\frac{M-1}{\tau_{e}}\right)\right] \\
& \times \frac{\sinh ^{2} \frac{t_{0}}{2 \tau_{w}} \sinh M \frac{t_{0}}{\tau_{e}}}{\sinh \frac{t_{0}}{\tau_{e}}} \\
\approx & \eta_{0} \frac{\tau_{e}{ }^{2}\left(1-e^{-2}\right)}{2 M^{2} \tau_{w}{ }^{2}} .
\end{aligned}
$$

Approximation (27) results from Eq. (26) if we substitute the optimal value of $t_{0}$ calculated in Eq. (23). Thus, in the sequential schedule, the average diffraction efficiency follows the $1 / M^{2}$ rule, but it is actually weaker than the diffraction efficiency of anglemultiplexed holograms by a factor of $\left(1-e^{-2}\right) / 2 \approx$ 0.432. On the other hand, from Eq. (26) we observe that if we let $t_{0} \rightarrow \infty, \eta_{\mathrm{av}}$ behaves like $1 / M$. This expresses the fact that if we overexpose the holograms in the sequential method, then only the first strip of each hologram will survive and the rest of the hologram will be erased. This situation is undesirable, as it restricts the recording area to a strip of width $\delta$ only and degenerates shift multiplexing to spatial multiplexing, resulting in severe losses in storage density.

At the leading edge, holograms $\mathrm{A}_{1}, \ldots, \mathrm{A}_{M}$ have uniform diffraction efficiency equal to $\eta^{M}$, because they receive additional exposure at the end of the schedule, when the disk is about to complete one revolution. At the trailing edge, the worst affected strip of hologram $\mathrm{A}_{N-m}, m=M-1, \ldots, 1$, is $l=$ $m+1$ and has a diffraction-efficiency value of $\eta^{l} \approx \eta_{0} \tau_{e}^{2} e^{-2(l-1) / M} / \tau_{w}^{2} M^{2}$. Hologram $\mathrm{A}_{N}$ is uniform, as it is never erased and has diffraction efficiency $\eta^{1}$.

We can cancel the nonuniformity in image-plane holograms by recording with the inverse intensity dependence. Alternatively, for digital storage, one can record uniform holograms and use variable decision thresholds. Either method will yield good results if the diffraction efficiency of the most affected areas is kept sufficiently strong compared with the noise level by the use of the optimal $t_{0}$ of Eq. (23). On the other hand, the nonuniformity has severe effects on Fourier holograms, as it shapes the hologram spectrum asymmetrically. This nonuniform filtering effect causes pixel broadening (intrapage noise); therefore the contrast ratio of the recon- 
struction decreases with respect to the unfiltered case. For holograms recorded in the Fresnel region, image- and Fourier-plane effects are combined in the sense that one observes nonuniformity across the reconstruction and also a decrease in the contrast ratio. In Appendix B we characterize the nonuniform erasure-induced filtering theoretically.

We used the sequential exposure schedule to record 600 holograms in the experimental setup shown in Fig. 3. We set the separation between adjacent holograms to $7.4 \mu \mathrm{m}$, which equals twice the measured shift selectivity $\delta=3.7 \mu \mathrm{m}$. The size of the signal beam projected onto the crystal surface was approximately $3 \mathrm{~mm}$. Therefore the number of overlapping holograms in this experiment was $M \approx$ 400. For the crystal we used and the given geometry, we measured $\tau_{e} \approx 3500 \mathrm{~s}$. For recording we used $t_{0}=10 \mathrm{~s}$ as the constant exposure time. Each reconstruction was spatially integrated onto a single detector in order to measure the diffraction efficiency. The results are plotted in Fig. 10. It is seen that the first 200 holograms were successfully equalized in terms of the total diffraction efficiency, as they all received equal exposure. From then on, the diffraction efficiency versus the hologram number attains an upward slope, as expected, because, as the order of holograms increases, the number of overlapping holograms decreases.

In Fig. 11 we show a few reconstructions from the 600 holograms. All holograms (with the exception of the last few) exhibit nonuniformity toward the shift direction. Because the image features were quite large in this experiment, the pixel-broadening effect was not observed.

We can eliminate the nonuniformity through the use of a different exposure schedule, which we call interleaved. With this scheme, we record one complete track of nonoverlapping (spatially multiplexed)

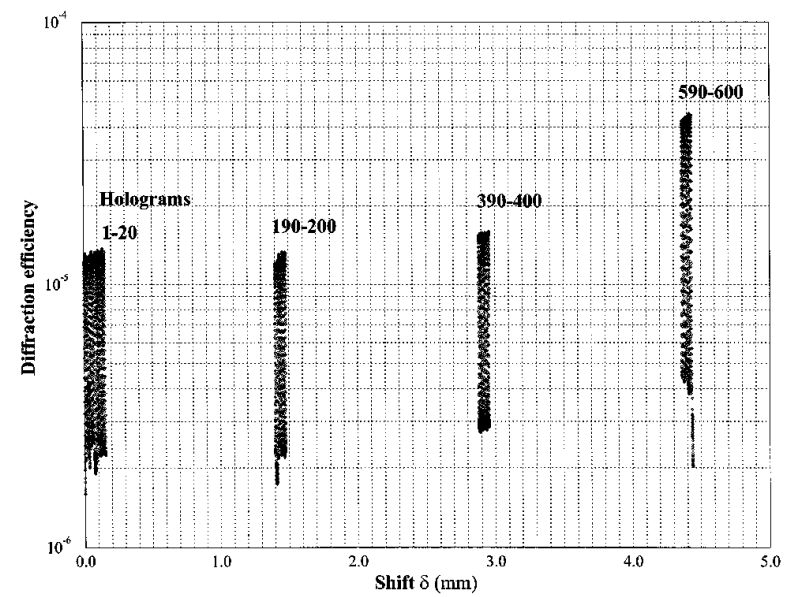

Fig. 10. Plot of the measured diffraction efficiency (after spatial integration by a single detector) of 50 out of 600 holograms stored with the sequential method. For a shift separation of $\delta_{\text {shift }}=7.4$ $\mu \mathrm{m}$ (second null) and an aperture size of $s \approx 3 \mathrm{~mm}$, we have $M \approx$ 400. Therefore only the first 200 holograms received equal exposure. The exposure time used in this experiment was $t_{0}=10 \mathrm{~s}$. holograms before moving to the next shift-multiplexed position. This method is well matched to the disk configuration, as we can record a new set of slightly shifted spatially multiplexed holograms during each disk rotation. Interleaving works perfectly if $N+1$ is an integer multiple of $M$; otherwise the first $M$ and last $M$ holograms suffer from overexposure and underexposure, respectively, as in the sequential method. We ignore these edge effects in the subsequent analysis.

As described, recording consists of $M$ epochs. At epoch $q(q=0, \ldots, M-1)$ we record holograms $\mathrm{A}_{q}$, $\mathrm{A}_{M+q}, \ldots, \mathrm{A}_{N-M+q}$. The recording time for all holograms at epoch $q$ is $t_{q}$. Because full tracks are recorded so that they completely overlap (but still they are displaced by $\delta$ with respect to each other), all holograms are erased uniformly; moreover, tracks recorded later are erased less than their predecessors. The uniform diffraction efficiency of the holograms after epoch $q$ is recorded is given by

$$
\eta(q)=\eta_{0}\left[1-\exp \left(-\frac{t_{q}}{\tau_{w}}\right)\right]^{2} \exp \left(\sum_{q^{\prime}=q+1}^{M-1} \frac{2 t_{q^{\prime}}}{\tau_{w}}\right) .
$$

This same equation holds for methods of complete overlap, e.g., angle multiplexing. ${ }^{15}$ Therefore the exposure schedule is determined identically. The optimal diffraction efficiency is given by

$$
\eta \approx \eta_{0} \frac{\tau_{e}^{2}}{M^{2} \tau_{w}^{2}}
$$

It is the same for all holograms and equal to the diffraction efficiency yielded by the exposure schedule for angle-multiplexed holograms. The price to pay for the equalization provided by the interleaving method is considerable complication in the recording process.

\section{Surface Storage Density}

The surface storage density of a holographic disk is defined ${ }^{3}$ as the number of bits of information (in the form of binary pixels) that are stored per unit area. Data are stored so that every page, which contains $N_{p} \times N_{p}$ pixels, $\left(N_{p}\right.$ per dimension) occupies area $\mathscr{b}$ on the disk. In volume holographic memories, the page density is multiplied by $M$, the number of overlapping holograms per location. Therefore the surface storage density $\mathscr{D}$ of any holographic disk is

$$
\mathscr{D}=\frac{M N_{p}^{2}}{\mathscr{C}} .
$$

The storage densities for angle- and wavelengthmultiplexed disks were calculated and optimized in Ref. 3. In this section we do the analogous calculation for shift-multiplexed disks when a spherical wave is used as reference.

First we consider the case in which holograms are stored in the image plane. Specifically, we assume 


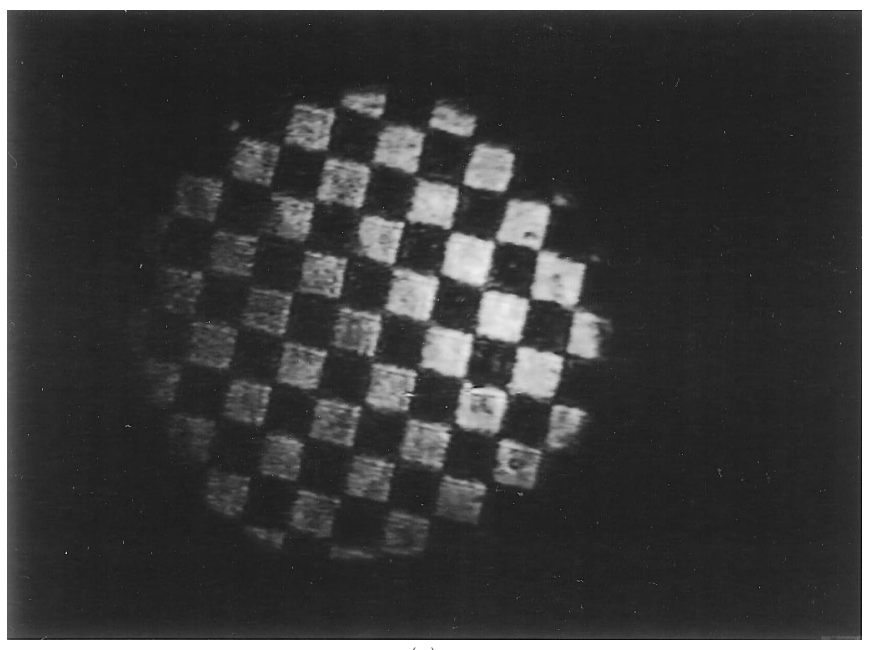

(a)

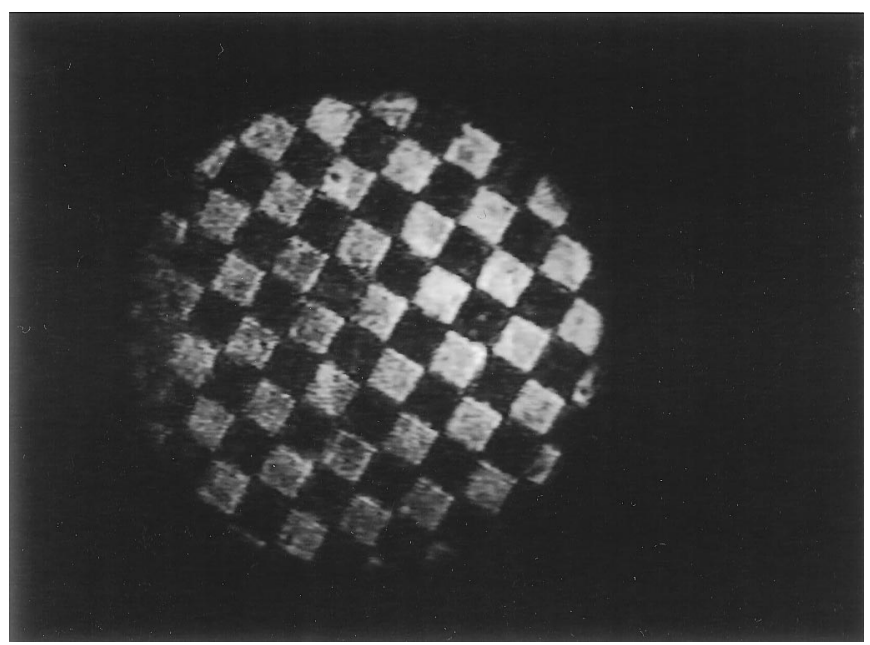

(b)

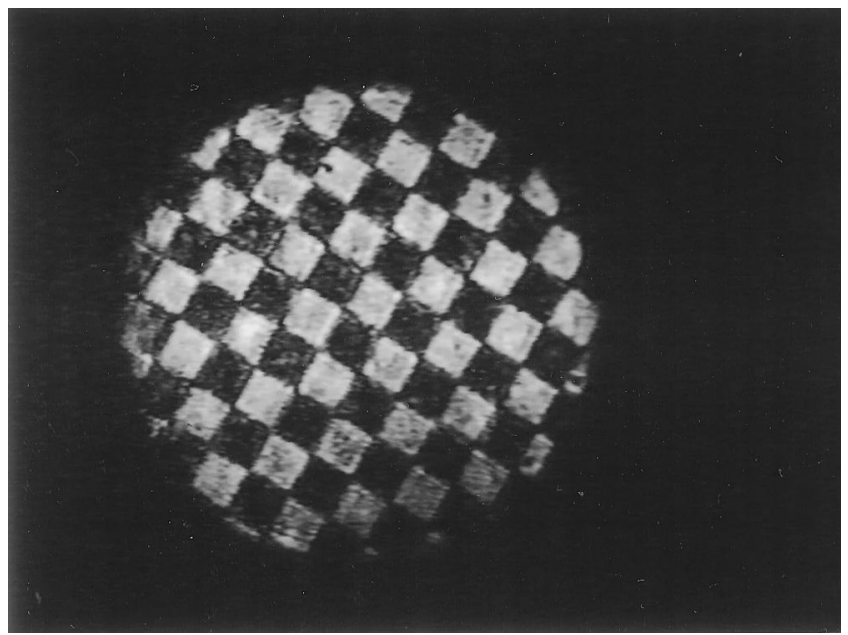

(c)

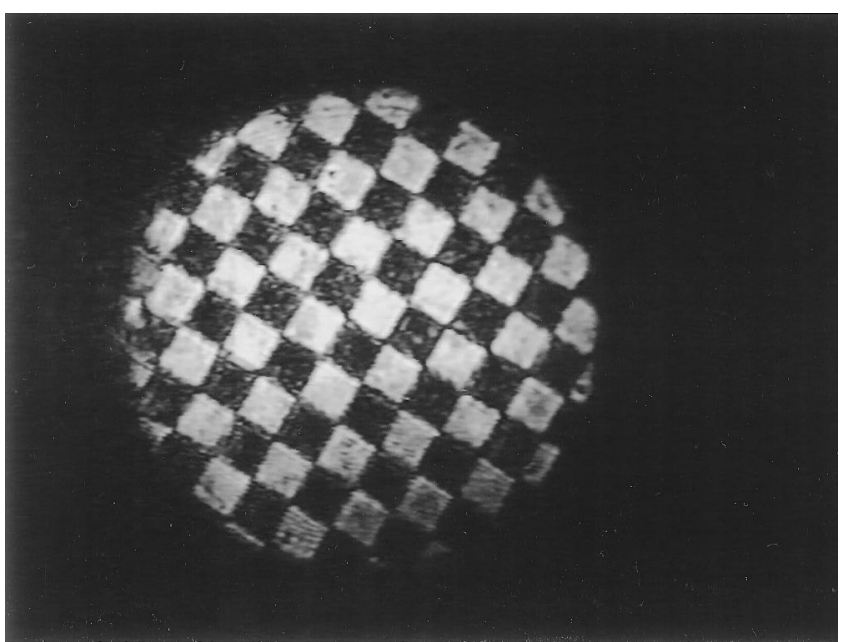

(d)

Fig. 11. Reconstructions of holograms (a) 1, (b) 200, (c) 400, and (d) 600 from the experiment of Fig. 10 . The shift direction was from left to right.

that the central pixel of the stored page is imaged at the center of the holographic medium. We denote by $b$ the size of the pixels in the image. Then the area is $\mathscr{b}=\left(N_{p} b\right)^{2} / \cos \theta_{S}$, where $\theta_{S}$ is the angle of incidence of the central signal component, as in the sections above. The number of overlapping shiftmultiplexed holograms along a single page is $M=$ $N_{p} b / 2 \delta \cos \theta_{S}$, where $\delta$ is the shift selectivity given by Eq. (8), and we assume that successive holograms are stored at the second Bragg null; this was justified in Section 3. Therefore we obtain, for the density,

$$
\mathscr{D}_{\text {image }}=\frac{N_{p}}{2 b \delta}=\frac{N_{p}}{2 b \lambda\left[\frac{z_{0}}{L \tan \theta_{S}}+\frac{1}{2(\mathrm{NA})}\right]} .
$$

For Fourier-plane storage, the size of the first lobe (which contains all the information, according to the sampling theorem) is $2 \lambda F / b$, where $F$ is the focal length of the Fourier-transforming lens and $b$ is the pixel size. The lobe size was derived assuming intensity detection. The result for the density is

$$
\mathscr{V}_{\text {Fourier }}=\frac{N_{p}{ }^{2} b}{4 \lambda F \delta}=\frac{N_{p}{ }^{2} b}{4 \lambda^{2} F\left[\frac{z_{0}}{L \tan \theta_{S}}+\frac{1}{2(\mathrm{NA})}\right]} .
$$

Equations (31) and (32) give the density, provided that the distance $z_{0}$ has been already selected properly such that the reference and the signal completely overlap inside the volume of the recording material. In general, the minimum $z_{0}$ is determined in terms of the hologram thickness and the geometry. We show that $z_{0}$ varies linearly with $L$, according to the relation

$$
z_{0}(L)=A+B L .
$$

Increasing the thickness beyond a certain point does not lead to the expected gain in density, because the reduction in Bragg selectivity that is due to the increased interaction length competes with the simul- 
taneous increase in $z_{0}$. Below we derive the coefficients $A$ and $B$ of Eq. (33) and the maximum achievable density with optimally selected $z_{0}$ as functions of thickness $L$.

We use $\phi$ for the angular spread of the reference beam, i.e., $\mathrm{NA}=\sin \phi$. We do the calculation simultaneously for the image and the Fourier planes. For this reason, we use the symbol $s$ for the page size in both cases, given respectively by

$$
s=\left\{\begin{array}{ll}
N_{p} b & \text { image plane } \\
2 \lambda_{0} F / b & \text { Fourier plane }
\end{array},\right.
$$

where $\lambda_{0}$ is the wavelength of light in vacuum. The angular spread of the signal beam outside the holographic material is

$$
\sin \chi \approx\left\{\begin{array}{ll}
\lambda_{0} / b & \text { image plane } \\
N_{p} b / 2 F, & \text { Fourier plane }
\end{array} .\right.
$$

Let $n_{0}$ denote the refractive index of the holographic material. The reference spread $\phi^{\prime}$, the angle of signal incidence $\theta_{S}{ }^{\prime}$, the signal spread $\chi^{\prime}$, and the page size $s^{\prime}$ inside the material are recalculated with Snell's law as follows:

$$
\begin{aligned}
\sin \phi & =n_{0} \sin \phi^{\prime}, \\
\sin \theta_{S} & =n_{0} \sin \theta_{S^{\prime}}, \\
\sin \chi & =n_{0} \sin \chi^{\prime}, \\
s \cos \theta_{S^{\prime}} & =s^{\prime} \cos \theta_{S} .
\end{aligned}
$$

Because the signal beam is tilted with respect to the normal to the recording material, it is possible that the tilted image of the data page does not fit inside the medium. This will happen if the medium is very thin (see details below) or if the tilt is large

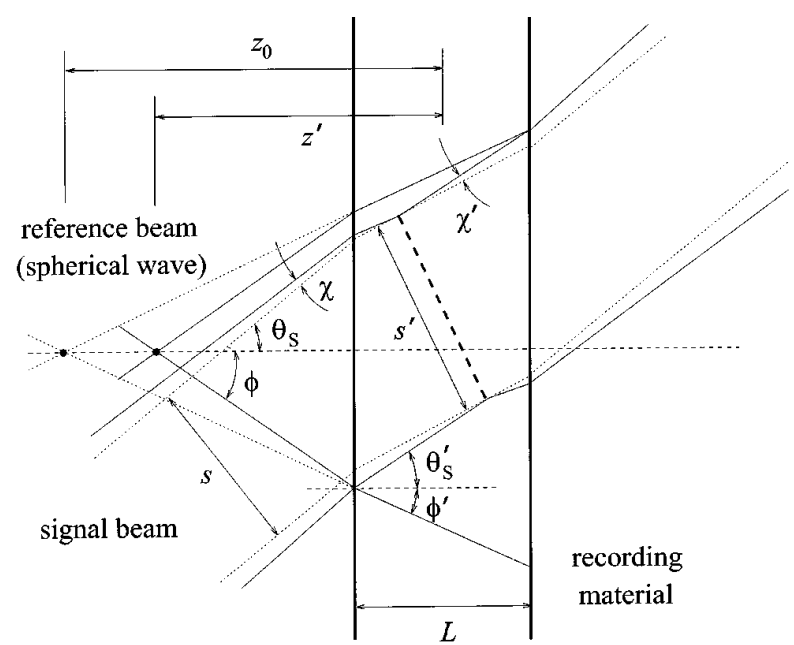

Fig. 12. Geometry for the calculation of storage density in shift-multiplexing geometry (spherical reference incident normally upon the material, signal incident off axis). The case $s^{\prime} \sin \theta_{S}^{\prime}<L, \phi<\theta_{S}$ is shown (see text). enough. Therefore we need to consider two separate cases for thick and thin media. We start with the case of a thick medium so that the condition $s^{\prime} \sin \theta_{S}{ }^{\prime}<L$ is satisfied, i.e., the whole focused page fits inside the hologram, as shown in Fig. 12. The geometry we chose for this analysis is conservative in the sense that we restricted the reference aperture according to $\phi<\theta_{S}$. This guarantees that the signal eventually separates itself from the reference cone, and thus the design of the imaging system that delivers the signal to the hologram is simplified. This restriction could be relaxed and the density would increase, but the optical design would become more complicated. We do not consider this optimization problem in this paper.

A geometric calculation based on Fig. 12 shows that the minimum $z_{0}$ required for the reference and the signal to overlap is given by Eq. (33) with coefficients

$$
\begin{aligned}
A & =\frac{s}{2} \frac{\cos \chi^{\prime} \cos \theta_{S^{\prime}}}{\cos \left(\theta_{S^{\prime}}+\chi^{\prime}\right) \tan \phi^{\prime} \cos \theta_{S}}, \\
B & =\frac{1}{2} \frac{\tan \left(\theta_{S^{\prime}}+\chi^{\prime}\right)}{\tan \phi^{\prime}} .
\end{aligned}
$$

Recall that $z_{0}$ is the apparent focal distance of the spherical wave, as seen by an observer inside the holographic medium. In order to convert $z_{0}$ to $z_{a}$ (focal distance measured in air), we apply Eq. (9). In the case of a thick medium, $z_{0}$ always increases with $L$, and the surface density saturates to

$$
\mathscr{D}_{\text {image }}^{\max }=\frac{n_{0} N_{p} \sin \phi^{\prime}}{\lambda_{0} b\left[1+\frac{\tan \left(\theta_{S}^{\prime}+\chi^{\prime}\right) \cos \phi^{\prime}}{\tan \theta_{S^{\prime}}}\right]}
$$

for the image plane and to

$$
\mathscr{D}_{\text {Fourier }}^{\max }=\frac{n_{0} N_{p}{ }^{2} b \sin \phi^{\prime}}{2 \lambda_{0}^{2} F\left[1+\frac{\tan \left(\theta_{S^{\prime}}+\chi^{\prime}\right) \cos \phi^{\prime}}{\tan \theta_{S^{\prime}}}\right]}
$$

for the Fourier plane.

For the case $s^{\prime} \sin \theta_{S}^{\prime}>L$ (thin medium), the geometric calculation is more complicated. With the same restrictions as above, the result is

$$
\begin{aligned}
A= & \frac{s}{2} \frac{\cos \chi}{\tan \phi^{\prime} \cos \left(\theta_{S}+\chi\right)}, \\
B= & \frac{1}{2 \tan \phi^{\prime}}\left[\frac{1}{\tan \theta_{S^{\prime}}}+2 \tan \left(\theta_{S^{\prime}}+\chi^{\prime}\right)\right. \\
& \left.-\frac{\cos \theta_{S} \cos \chi}{\sin \theta_{S^{\prime}} \cos \theta_{S}{ }^{\prime} \cos \left(\theta_{S}+\chi\right)}\right] .
\end{aligned}
$$

Note that the coefficient $B$ for a thin medium can become negative, and then the optimal $z_{0}$ decreases 
with $L$. This is due to bending of the signal rays induced by refraction.

In a recent experiment, ${ }^{17}$ surface storage density in excess of $10 \mathrm{bits} / \mu^{2}$ with a raw bit-error rate of $10^{-4}$ was demonstrated in a holographic disk configuration with DuPont's HRF-150-100 photopolymer as the recording material. The parameters used in this experiment were $\lambda=0.532 \mathrm{~nm}, n_{0}=1.525, N_{p}=$ $768, b=45 \mu \mathrm{m}$, and $F=5.46 \mathrm{~cm}$. Thirty-two Fresnel-region holograms were superimposed on the same spot by a combination of angle (eight locations separated by four Bragg nulls) and peristrophic ${ }^{18}$ (four holograms per angular location) multiplexing.

Shift multiplexing can also be combined with other techniques, such as peristrophic and fractal, ${ }^{19}$ in order to increase the storage density at the cost of complicating page access. Better yet, it is possible to apply the spherical reference analog to the angle plus fractal or peristrophic methods, which consists of shift multiplexing holograms in both the $x$ and $y$ directions (see Fig. 2). In the disk configuration, $y$-shift multiplexing corresponds to overlapping hologram tracks. The $y$-shift selectivity for high-bandwidth signal beams is given by ${ }^{20}$

$$
\delta_{y}=z_{0}\left(\frac{2 \lambda}{L}\right)^{1 / 2}+\frac{\lambda}{2(\mathrm{NA})} .
$$

For the same parameters of the experiment of Ref. 17, $y$-shift multiplexing increases the density by a factor of at least 3.

Using the combination of $y$-shift multiplexing with $x$-shift multiplexing at the fourth shift Bragg null (consistent with Ref. 17), $\phi=45^{\circ}, \theta_{S}=60^{\circ}$, and assuming Fourier-plane storage, we obtain the theoretical density prediction for shift multiplexing given in Fig. 13. Note that, for thickness $L=100 \mu \mathrm{m}$ of the DuPont photopolymer, $x+y$-shift multiplexing is expected to yield $\mathscr{D}=11.8 \mathrm{bits} / \mu^{2}$, slightly higher than the $10.7 \mathrm{bits} / \mu_{\mathrm{m}}^{2}$ of the high-density experiment reported in Ref. 17. Shift density increases almost linearly with thickness, reaching 163.4 $\mathrm{bits} / \mu^{2}$ for $L=1.2 \mathrm{~mm}$, when it begins to saturate. Thus shift multiplexing utilizes the area of holographic 3-D disks more efficiently.

\section{Discussion and Conclusions}

In this paper we analyzed theoretically and experimentally shift multiplexing by using spherical reference waves. We addressed the issues of selectivity, cross talk, exposure schedule, and storage density. We showed the similarity between the Bragg selectivity mechanisms of angle and shift multiplexing and pointed out the differences that permit shift multiplexing to achieve higher storage density in a more compact setup.

Variants of the shift-multiplexing method described herein are also possible. For example, one could relax the constraint of the signal off-axis geometry and use the signal on axis with a tilted

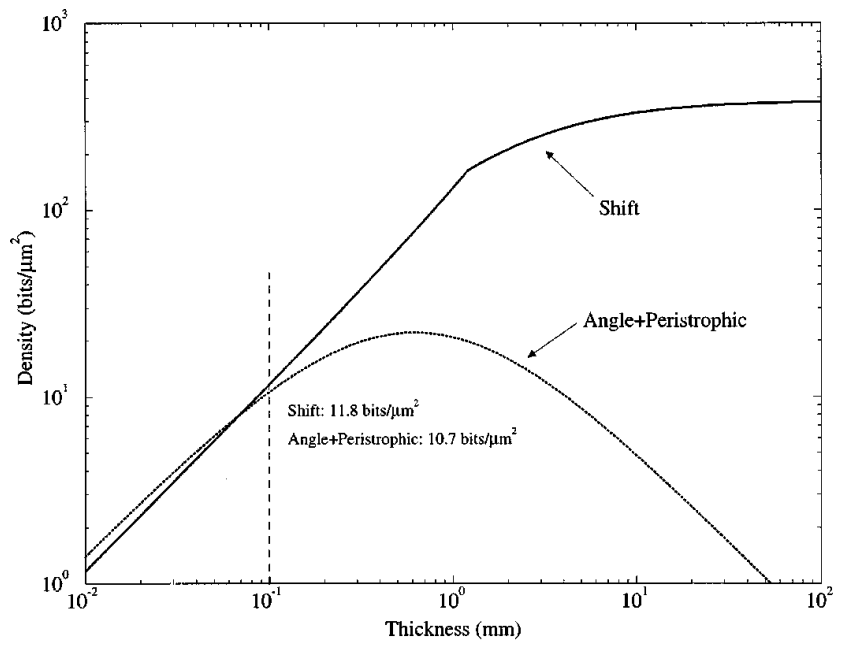

Fig. 13. Theoretical shift-multiplexing surface storage density in the Fourier plane, with parameters $\lambda=0.532 \mathrm{~nm}, n_{0}=1.525$, $N_{p}=768, b=45 \mu \mathrm{m}$, amd $F=5.46 \mathrm{~cm}$, the same as those of the experiment reported in Ref. 17, where angle plus peristrophic multiplexing was used. The reference spread used for the shiftmultiplexing density calculation is $\phi=45^{\circ}$, and the angle of incidence of the signal beam is $\theta_{S}=60^{\circ}$.

spherical wave of small NA as reference, even though in such a system the shift selectivity would be significantly worse. Shift multplexing can also be implemented in the reflection and $90^{\circ}$ geometries. In particular, the $90^{\circ}$ geometry yields optimal selectivity

$$
\delta=\frac{\lambda z_{0}}{2 L}+\frac{\lambda}{2(\mathrm{NA})}
$$

[in agreement with Eq. (7)]. However, for storage in photorefractives, the transverse nonuniform erasure effect analyzed for transmission geometry in Appendix B can be shown to broaden the selectivity in that case. This leads to higher cross talk, as the hologram acquires an intensity profile along the $z$ direction, and therefore the achievable storage density is reduced.

In this paper we concentrated on shift multiplexing in the $x$ direction (parallel to the plane defined by the direction of the signal beam and the point source). The possibility of shift multiplexing in the $y$ direction ${ }^{20}$ was mentioned in Section 5. In the holographic disk architecture, $y$-shift multiplexing is achieved by translation of the head with the spherical reference in the radial direction with respect to the disk. Then the reconstruction also shifts in the detector plane, until eventually it misses the detector or becomes Bragg mismatched. The required shift distance before either effect occurs is typically a few hundred micrometers. One can record a new track, overlapping with the previous one, on the new location by multiplexing in the $x$ direction. The gain in density can be of the order of $2 \sim 10$, depending on the signal bandwidth and the focal distance of the spherical reference. 
Appendix A. Derivation of the Shift Selectivity

In this appendix we derive the diffraction efficiency of a spherical volume hologram as a function of the shift $\delta$ of the reference relative to the hologram. We consider again the geometry of Fig. 2. Under the Born approximation, the diffracted field at the observation point $\mathbf{r}_{p}$ is given by the volume integral, ${ }^{21}$

$$
\mathscr{E}_{d}\left(\mathbf{r}_{p}\right)=\int_{\mathscr{T}} \mathscr{E}_{i}(\mathbf{r}) \Delta \epsilon(\mathbf{r}) G\left(\mathbf{r} ; \mathbf{r}_{p}\right) \mathrm{d}^{3} \mathbf{r}
$$

where $\mathscr{T}$ denotes the volume of the hologram, $\mathscr{E}_{i}$ is the incident field, $\Delta \epsilon(\mathbf{r})$ is the phase hologram, and $G\left(\mathbf{r} ; \mathbf{r}_{p}\right)$ is the scalar Green's function for free space ${ }^{22}$ :

$$
\begin{aligned}
G\left(\mathbf{r} ; \mathbf{r}_{p}\right)= & \frac{1}{j \lambda\left|\mathbf{r}-\mathbf{r}_{p}\right|} \exp \left(j 2 \pi \frac{\left|\mathbf{r}-\mathbf{r}_{p}\right|}{\lambda}\right) \\
\approx & \frac{1}{j \lambda\left(z_{p}-z\right)} \exp \left[j 2 \pi \frac{z_{p}-z}{\lambda}\right. \\
& \left.+j \pi \frac{\left(x_{p}-x\right)^{2}+\left(y_{p}-y\right)^{2}}{\lambda\left(z_{p}-z\right)}\right],
\end{aligned}
$$

We obtain the last relation by expressing a spherical wave in the paraxial approximation. We assumed that $z_{p}>z$ for all pairs of observation-integration points $z_{p}, z$.

Similarly, the spherical reference wave (on recording) is expressed in the paraxial approximation as in Eq. (1), repeated here for convenience,

$$
\begin{aligned}
R(\mathbf{r})= & \frac{1}{j \lambda\left(z+z_{0}\right)} \exp \left(j 2 \pi \frac{z+z_{0}}{\lambda}\right) \\
& \times \exp \left[j \pi \frac{x^{2}+y^{2}}{\lambda\left(z+z_{0}\right)}\right]
\end{aligned}
$$

and the propagating signal is expressed as

$$
S(\mathbf{r})=\exp \left(j 2 \pi u_{S} \frac{x}{\lambda}\right) \exp \left[j 2 \pi\left(1-\frac{u_{S}^{2}}{2}\right) \frac{z}{\lambda}\right] .
$$

Then we have

$$
\begin{aligned}
& \Delta \epsilon(\mathbf{r})=R^{*}(x, y, z) S(x, z), \\
& \mathscr{E}_{i}(\mathbf{r})=R(x-\delta, y, z) .
\end{aligned}
$$

We also assume that the spherical wave and the recording material are infinite in the transverse $(x, y)$ directions and that the thickness of the hologram is $L$ in the $z$ direction. Substituting into Eq. (A1), we obtain the following expression:

$$
\mathscr{E}_{d}\left(\mathbf{r}_{p}\right) \approx \frac{\exp \left(j 2 \pi \frac{z_{p}}{\lambda}\right)}{\lambda^{2} z_{0}^{2}} \int_{-\infty}^{+\infty} \mathrm{d} x \int_{-\infty}^{+\infty} \mathrm{d} y \int_{-\infty}^{+\infty} \mathrm{d} z
$$

$$
\begin{aligned}
& \times \operatorname{rect}\left(\frac{z}{L}\right) \exp \left[-j \pi\left(u_{S}{ }^{2} \frac{z}{\lambda}-\frac{\delta^{2}}{\lambda\left(z+z_{0}\right)}\right)\right] \\
& \times \exp \left[j 2 \pi \frac{x}{\lambda}\left(u_{S}-\frac{\delta}{z_{0}+z}\right)\right] \\
& \times \frac{1}{\lambda\left(z_{p}-z\right)} \exp \left(j 2 \pi \frac{z_{p}-z}{\lambda}\right) \\
& \times \exp \left[j \pi \frac{\left(x_{p}-x\right)^{2}+\left(y_{p}-y\right)^{2}}{\lambda\left(z_{p}-z\right)}\right] .
\end{aligned}
$$

The volume integral is calculated analytically as follows: The $x$ and the $y$ integrals are readily obtained with the following lemma from complex analysis ${ }^{23}$ :

$$
\begin{aligned}
\int_{-\infty}^{+\infty} \exp \left[j \left(a w^{2}+\right.\right. & 2 b w)] \mathrm{d} w \\
& =\left(\frac{\pi}{\mid a}\right)^{1 / 2} \exp \left[j\left(\operatorname{sgn}(a) \frac{\pi}{4}-\frac{b^{2}}{a}\right)\right],
\end{aligned}
$$

for $a, b$ real, and $a \neq 0$. Then we expand the denominators of the form $\left(z+z_{0}\right)^{m},(m=1,2)$ in the exponents, keeping terms of order $\left(z / z_{0}\right)$ only. The resulting $z$ integral yields

$$
\begin{array}{r}
\approx \frac{\exp \left\{j \frac{2 \pi}{\lambda}\left[u_{S} x_{p}+\left(1-\frac{u_{S}}{2}\right) z_{p}\right]+j \frac{2 \pi}{\lambda} \frac{\delta\left(x_{p}-u_{S} z_{p}\right)}{z_{0}}\right\}}{\lambda^{2} z_{0}^{2}} \\
\times \operatorname{sinc}\left[\frac{\delta L}{\lambda z_{0}}\left(u_{S}-\frac{x_{p}-u_{S} z_{p}}{z_{0}}\right)\right] \cdot \quad \text { (A9) }
\end{array}
$$

The first term in approximation (A9) is explained as follows: if $\delta=0$, the diffracted far field is a plane wave propagating in the direction $u_{S}$ of the original signal. For $\delta \neq 0$, the direction of the reconstruction deviates by $\delta / z_{0}$ (paraxially) from $u_{S}$. The direction-dependent sinc term suppresses the diffracted power, a result of phase mismatch among wavelets produced in different positions along the volume hologram (Bragg mismatch). In the far field we can make the stationary phase assumption (i.e., assume that significant diffraction is obtained only at $x_{p} \approx$ $\left.u_{S} z_{p}\right)$ to obtain, for the diffraction efficiency,

$$
\eta(\delta) \equiv \frac{\left|\mathscr{E}_{d}\right|^{2}}{\left|\mathscr{E}_{i}\right|^{2}} \sim \operatorname{sinc}^{2}\left(\frac{\delta u_{S} L}{\lambda z_{0}}\right) .
$$

Therefore, under the above assumptions, the Bragg nulls in diffraction efficiency occur at

$$
\delta=m \delta_{\mathrm{Bragg}} \equiv m \frac{\lambda z_{0}}{L u_{S}}, \quad m=1,2, \ldots
$$


Appendix B. Filtering Effects Induced by Nonuniform Erasure in the Fresnel and the Fourier Regimes

Shift-multiplexed holograms in photorefractive crystals stored according to the sequential schedule suffer nonuniform erasure (see Section 4). We now characterize this effect for holograms stored in the Fresnel region and in the Fourier plane as a special case.

The geometry used for the calculation is shown in Fig. 14. The hologram is tilted with respect to the

$$
\begin{aligned}
\beta(x) & =\cos \theta_{S}+\frac{x}{F} \sin \theta_{S}, \\
m_{0}(x) & =\frac{2 \lambda F}{b \beta(x) \delta} .
\end{aligned}
$$

The total number of strips $M$ is needed in order to determine the optimum recording time according to the theory of Section $4 . \quad M$ depends on the defocusing distance $f$ and is given by

$$
M(f)=\left\{\begin{array}{ll}
\frac{2 \lambda F}{b \delta \cos \theta_{S}}\left(1+\left|1-\frac{f}{F}\right| \frac{N_{p} b^{2}}{2 \lambda F}\right), & \text { if }|F-f|>\frac{\lambda F}{b} \tan \theta_{S} \\
\frac{2 \lambda F}{b \delta \cos \theta_{S}}\left(1+\frac{N_{p} b}{2 F} \tan \theta_{S}\right), & \text { if }|F-f|<\frac{\lambda F}{b} \tan \theta_{S}
\end{array} .\right.
$$

signal beam path by angle $\theta_{S}$ and is located distance $f$ from the Fourier-transforming lens (focal length $F$ ). For simplicity we ignore the thickness of the recording material and the possible aberrations introduced by the tilted path. The shift selectivity is $\delta$, and the pixel size is $b$. We assume that during recording, the signal is low-pass filtered at the Nyquist cutoff bandwidth $2 \lambda F / b$ so that the area it takes on the disk is minimized without any loss in information content. Because of the shift-multiplexing mechanism, successive slices of the hologram suffer exponential erasure by an amount $t_{0}$ compared with that of their neighbors. Thus the diffraction efficiency is given by the staircaselike function,

$$
\begin{aligned}
\eta\left(x^{\prime \prime}, x\right)= & \sum_{l=0}^{m_{0}-1} \exp \left(-j 2 \pi \frac{x x^{\prime \prime}}{\lambda F}\right) \exp \left[-\left(l_{0}+l\right) t_{0} / \tau_{e}\right] \\
& \times \operatorname{rect}\left[\frac{x^{\prime \prime}-\left(l-\frac{m_{0}-1}{2}\right) \beta \delta}{\beta \delta}\right],
\end{aligned}
$$

where the indices $l_{0}$ and $m_{0}$ indicate which part of the staircase corresponds to the point source located at $x$ and $\beta$ is a correction factor for the tilt. These parameters are obtained directly from the geometry of Fig. 14, and are given by the following expressions:

$$
\begin{aligned}
& l_{0}(x, f)=\left|F-f-\frac{\lambda F}{b} \tan \theta_{S}\right|
\end{aligned}
$$

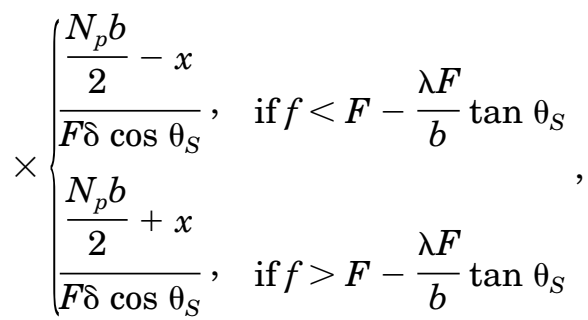

The transfer function is then determined as

$$
\begin{aligned}
h\left(x^{\prime}, x\right)= & \operatorname{sinc}(\kappa) \operatorname{ar}\left(\kappa+j \zeta ; m_{0}\right) \\
& \times \exp \left[-2 \pi\left(l_{0}+\frac{m_{0}-1}{2}\right) \zeta,\right. \\
\kappa= & \frac{\left(x^{\prime}-x\right) \beta(x) \delta}{\lambda F}, \\
\zeta= & \frac{t_{0}}{2 \pi \tau_{e}},
\end{aligned}
$$

where the array function $\operatorname{ar}(u ; l)$ is defined as

$$
\operatorname{ar}(u ; l)=\frac{\sin (l \pi u)}{l \sin (\pi u)} .
$$

Note that the filter represented by this transfer function is shift variant (unless $f=F$ and $\theta_{S}=0$, which would yield bad shift selectivity). In general, the diffraction efficiency is asymmetric (except when $f=F$ ), in agreement with experiment (see Fig. 11). The weaker edge is toward the shift direction if $f<F$ and in the opposite direction otherwise. The resolution is worse than the case of no erasure $\left(\tau_{e}=\infty\right)$ and decreases uniformly toward the weaker edges.

Some sample simulated reconstructions are shown in Fig. 15. The parameters used for this numerical example were $\lambda=488 \mathrm{~nm}, F=5 \mathrm{~cm}, N_{p}=10, b=$ $100 \mu \mathrm{m}, \theta_{S}=40^{\circ}$, and $\delta=7 \mu \mathrm{m}$. The original pattern used for the simulations is shown in Fig. 15(a). In Fig. 15(b) we have plotted the reconstruction for $f=4 \mathrm{~cm}$ with no absorption $\left(t_{0} / \tau_{e}=0\right)$. In this case simple low-pass filtering takes place, with a cutoff frequency equal to the Nyquist frequency $2 \lambda F / b$ determined for intensity detection. The contrast ratio is $\mu=91.41$ in this example. For a Fourier filter with $t_{0} / \tau_{e}=0.011$ (approximately equal 


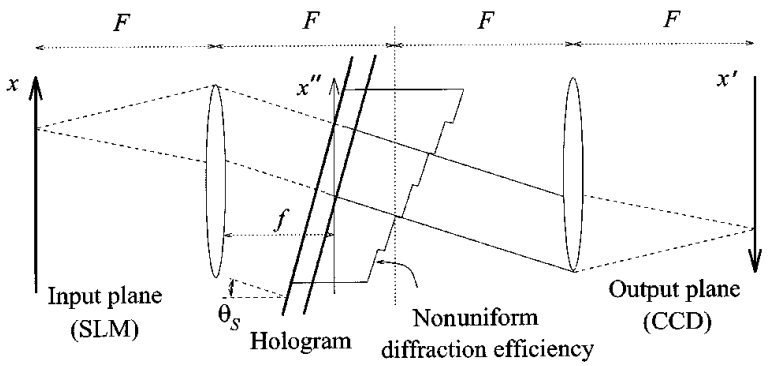

Fig. 14. Geometry for the calculation of the distortion occuring in shift-multiplexed holograms recorded in photorefractive materials, which is due to partial erasure in the Fourier or the Fresnel regions. The filter is shift variant if the hologram is not centered with respect to the Fourier plane (see also Fig. 9).
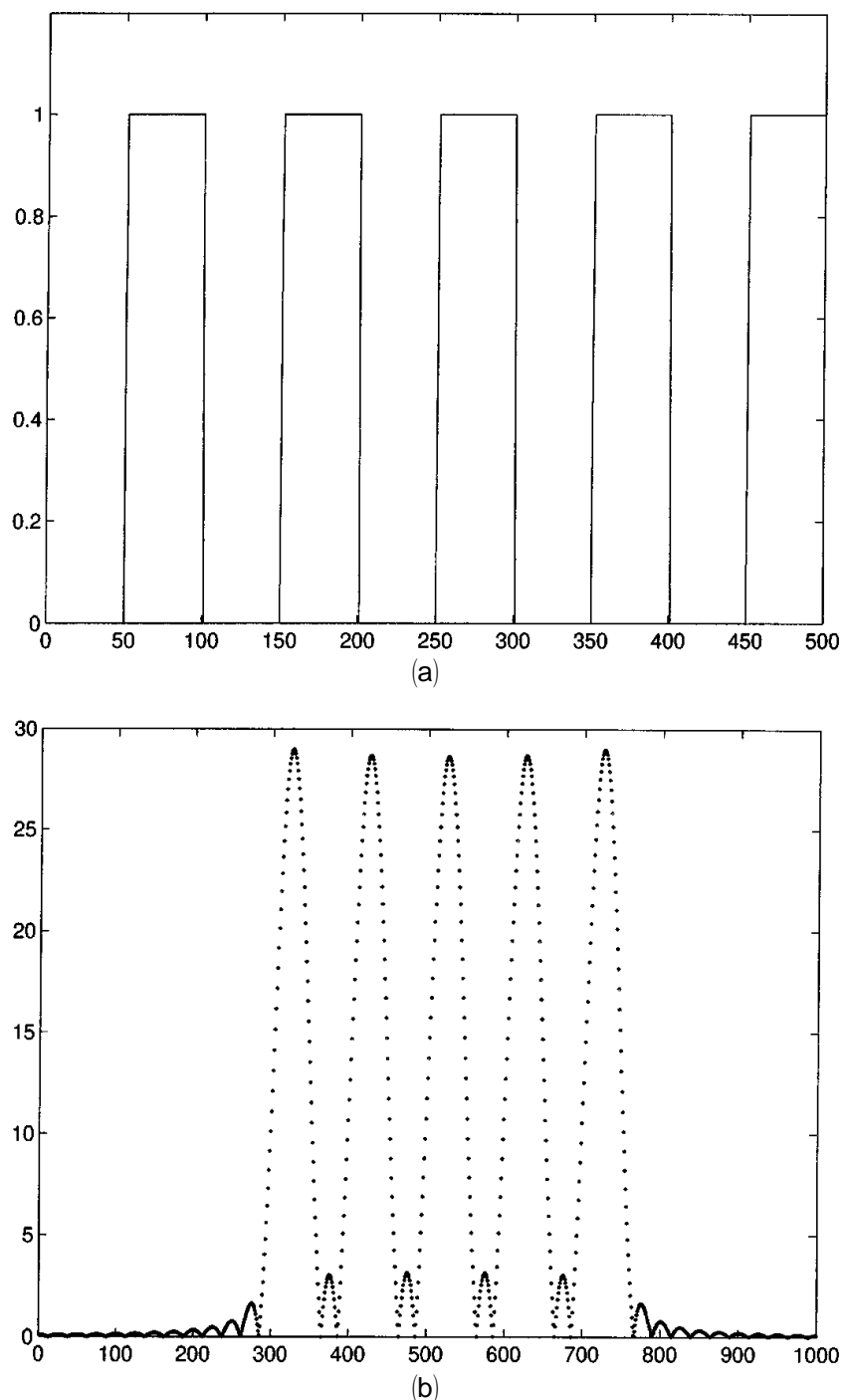

to $1 / M$, where $M=92$ for this case), Fig. $15(\mathrm{c})$, the contrast ratio drops to $\mu \approx 72,21.2 \%$ down with respect to the simple Nyquist filter. Finally, in Fig. 15(d) the result of a Fresnel filter with $f=4 \mathrm{~cm}, M=$ 109 , and $t_{0} / \tau_{e}=1 / M=0.0092$ are shown. The contrast ratio is $\mu=72.15$ at the weak edge and $\mu=$ 83.04 at the strong edge. Even though $\mu$ improved, the average diffraction efficiency $\eta_{\mathrm{av}}$ decreased according to the theory of Section 4 , since $M$ increased, and therefore other noise sources degrade the total SNR. If, however, we were to keep $t_{0} / \tau_{e}=0.011$ for the Fresnel filter, then $\eta_{\mathrm{av}}$ would improve, but $\mu$ would drop to 66.61 and 78.8 at the weak and the strong edges, respectively.

This research was supported by the U.S. Air Force Office of Scientific Research under grant F49620-92$\mathrm{J}-0400$. We are grateful to Allen Pu, Kevin Curtis, and Ajay Chugh for their assistance, to Geoffrey
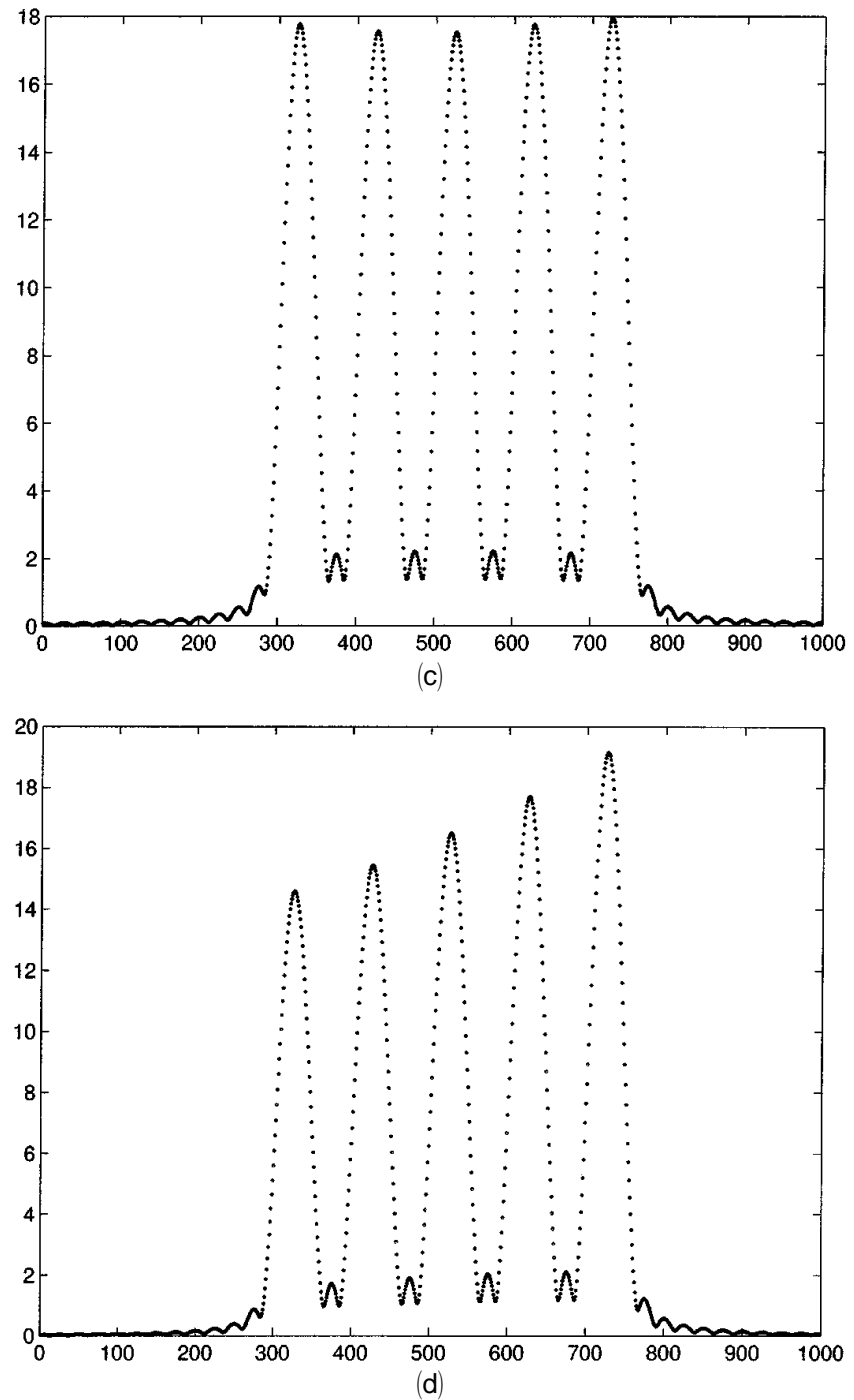

Fig. 15. Numerical results of the effects of shift-induced nonuniformity on Fourier and Fresnel holograms. The horizontal axis is the image coordinate parallel to the shift direction, and the vertical axis is diffracted power, both in arbitrary units. (a) Original chessboard pattern; (b) Nyquist filter (cutoff at $\pm \lambda F / b$ ) without absorption $\left(\tau_{e}=\infty\right)$, located at $f=F=5 \mathrm{~cm}$; (c) Nyquist filter with $t_{0} / \tau_{e}=0.011, f=F=$ $5 \mathrm{~cm}$ (Fourier filter); and (d) Nyquist filter with $t_{0} / \tau_{e}=0.0092, f=4 \mathrm{~cm}$ (Fresnel filter). 
Burr, Jean-Jacques Drolet, Fai Mok, and Gan Zhou for helpful discussions and suggestions, and to Yayun Liu for technical support. Part of this work was conducted while George Barbastathis was supported by a Charles Lee Powell Foundation graduate fellowship. Michael Levene acknowledges the support of a National Defence Science and Engineering Graduate fellowship.

\section{References}

1. D. Psaltis, M. Levene, A. Pu, G. Barbastathis, and K. Curtis, "Holographic storage using shift multiplexing," Opt. Lett. 20, 782-784 (1995).

2. D. Psaltis, "Parallel optical memories," Byte 17, 179-182 (1992).

3. H.-Y. S. Li and D. Psaltis, "Three-dimensional holographic disks," Appl. Opt. 33, 3764-3774 (1994).

4. K. Wagner and D. Psaltis, "Multilayer optical learning networks,"Appl. Opt. 26, 5061-5076 (1987).

5. L. Solymar and D. J. Cooke, Volume Holography and Volume Gratings (Academic, New York, 1991), pp. 243-253.

6. H. C. Külich, "A new approach to read volume holograms at different wavelengths," Opt. Commun. 64, 407-411 (1987).

7. H. C. Külich, "Reconstructing volume holograms without image field losses," Appl. Opt. 30, 2850-2857 (1991).

8. C. Gu, J. Hong, I. McMichael, R. Saxena, and F. Mok, "Cross-talk-limited storage capacity of volume holographic memory,” J. Opt. Soc. Am. A 9, 1978-1983 (1992).

9. A. Yariv, "Interpage and interpixel cross talk in orthogonal (wavelength-multiplexed) holograms," Opt. Lett. 18, 652-654 (1993).

10. K. Curtis, C. Gu, and D. Psaltis, "Cross talk in wavelengthmultiplexed holographic memories," Opt. Lett. 18, 1001-1003 (1993).
11. K. Curtis and D. Psaltis, "Cross talk in phase-coded holographic memories," J. Opt. Soc. Am. A 10, 2547-2550 (1993).

12. K. Curtis and D. Psaltis, "Cross talk for angle- and wavelengthmultiplexed image plane holograms," Opt. Lett. 19, 17741776 (1994).

13. P. Yeh, Introduction to Photorefractive Nonlinear Optics (Wiley, New York, 1993), pp. 105-111.

14. K. Bløtekjaer, "Limitations on holographic storage capacity of photochromic and photorefractive media," Appl. Opt. 18, 57-67 (1979).

15. D. Psaltis, D. Brady, and K. Wagner, "Adaptive optical networks using photorefractive crystals," Appl. Opt. 27, 17521759 (1988).

16. E. S. Maniloff and K. M. Johnson, "Maximized photorefractive data storage," J. Appl. Phys. 70, 4702-4707 (1991).

17. A. Pu and D. Psaltis, "High-density recording in photopolymerbased holographic three-dimensional disks," Appl. Opt. 35, 2389-2398 (1996).

18. K. Curtis, A. Pu, and D. Psaltis, "Method for holographic storage using peristrophic multiplexing," Opt. Lett. 19, 993994 (1994).

19. F. H. Mok, G. W. Burr, and D. Psaltis, "Angle and space multiplexed random access memory (HRAM)," Opt. Mem. Neural Networks 3, 119-127 (1994).

20. A. Pu, G. Barbastathis, M. Levene, and D. Psaltis, "Shiftmultiplexed holographic 3D disk," in Optical Computing, Vol. 10 of 1995 OSA Technical Digest Series (Optical Society of America, Washington, D.C., 1995), pp. 219-221.

21. J. D. Jackson, Classical Electrodynamics, 2nd ed. (Wiley, New York, 1975), pp. 418-421.

22. J. W. Goodman, Introduction to Fourier Optics (McGraw-Hill, New York, 1968), pp. 33-39.

23. G. B. Whitham, Linear and Nonlinear Waves (Wiley-Interscience, New York, 1973), pp. 371-373. 